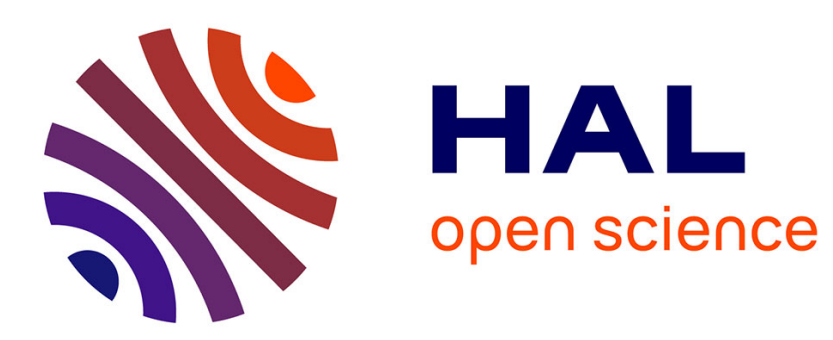

\title{
Régulateur de température transistorisé à effet continu pour microbalance à quartz vibrant
}

\author{
J. Gouault, M. Hubin, J.P. Leplat
}

\section{To cite this version:}

J. Gouault, M. Hubin, J.P. Leplat. Régulateur de température transistorisé à effet continu pour microbalance à quartz vibrant. Revue de Physique Appliquée, 1967, 2 (4), pp.289-291. 10.1051/rphysap:0196700204028900 . jpa-00242807

\section{HAL Id: jpa-00242807 https://hal.science/jpa-00242807}

Submitted on 1 Jan 1967

HAL is a multi-disciplinary open access archive for the deposit and dissemination of scientific research documents, whether they are published or not. The documents may come from teaching and research institutions in France or abroad, or from public or private research centers.
L'archive ouverte pluridisciplinaire HAL, est destinée au dépôt et à la diffusion de documents scientifiques de niveau recherche, publiés ou non, émanant des établissements d'enseignement et de recherche français ou étrangers, des laboratoires publics ou privés. 


\title{
RÉGULATEUR DE TEMPÉRATURE TRANSISTORISÉ A EFFET GONTINU POUR MIGROBALANGE A QUARTZ VIBRANT
}

\author{
Par J. GOUAULT, M. HUBIN et J. P. LEPLAT, \\ Institut National Supérieur de Chimie Industrielle de Rouen, 76-Mont-Saint-Aignan \\ et Faculté des Sciences de Rouen.
}

\begin{abstract}
Résumé. - Description et étude du fonctionnement d'une version transistorisée d'un dispositif à tubes déjà réalisé [3], destiné à régler d'une façon continue la température d'un quartz vibrant placé dans une enceinte à vide et utilisé comme microbalance [1].
\end{abstract}

\begin{abstract}
A transistorised version of a vacuum-tubes device already realised [3], in order to control in a continuous manner, the temperature of a vibrating quartz used as a vacuum microbalance for thin films, is described and studied.
\end{abstract}

Principe. - L'organe dont la température est réglée est un quartz vibrant $Q$ recevant des dépôts obtenus par vaporisation, dans le vide ou l'ultravide [1]. Sont associés à ce quartz : un système électronique qui le fait vibrer, un thermocouple $\mathrm{Ni}-\mathrm{Cu}$, dont la jonction chaude à la température $T_{q}$ est constituée par deux couches minces Ni-Gu déposées sur l'une de ses faces près d'un bord et dont la jonction froide est placée dans un bain à $0^{\circ} \mathrm{C}$, puis une résistance chauffante $\mathrm{R}$ située à proximité.

La fonction de transfert relative aux échanges de chaleur affectant le quartz vibrant est de la forme, en transformées de Laplace [5] :

$$
A(p) L \Delta T_{q}=a(p) L\left(\Delta R i^{2}\right)+L \Delta F_{c}
$$

$\Delta T_{q}, \Delta\left(R i^{2}\right), \Delta F_{c}$ étant respectivement les variations à partir de valeurs initiales, de la température du quartz, de la puissance fournie à la résistance chauffante et du flux thermique reçu par le quartz de la part du creuset évaporateur.

Le rôle du régulateur est de compenser toute variation du flux émis par le creuset par une variation de la puissance fournie au filament afin que $\Delta T_{q}$ varie entre des limites telles que la f.é.m. $e$ du thermocouple demeure égale à $e_{0}$ choisi à $\delta \mathrm{e}$ près ( $\delta \mathrm{e}$ de l'ordre de $2 \mu \mathrm{V}$, correspondant à $\Delta T_{q}$ égal à $0,1^{\circ} \mathrm{C}$ si le thermocouple $\mathrm{Ni}-\mathrm{Cu}$ était entièrement massif).

Description. - Le régulateur comprend les éléments suivants : $\mathrm{G}$, galvanomètre; $\mathrm{DG}$, double cellule insérée dans un pont alimenté par une tension sinusoïdale $V_{m} \cos \omega t$ de fréquence égale à celle du réseau; AS, amplificateur sélectif avec pont double en T; AP, amplificateur push-pull; $\mathrm{D}$, démodulateur; $\mathrm{M}$, servo-moteur à courant continu; $\mathrm{P}$, potentiomètre; $\mathrm{C}_{1}$, correcteur de phase; $\mathrm{C}_{2}$, convertisseur de puissance pour chauffage constitué par un transistor (fig. 1).

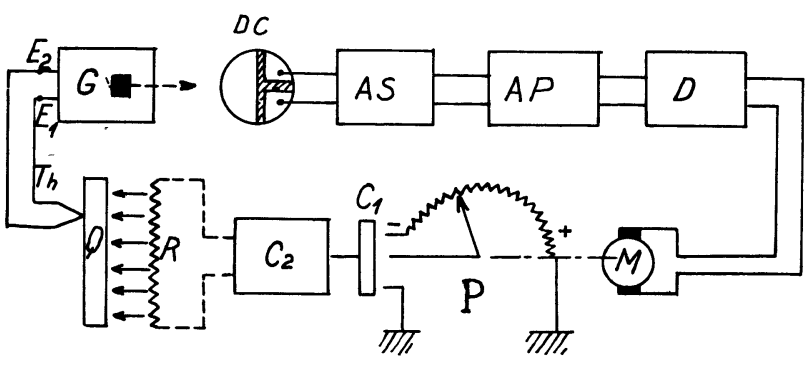

FIG. 1.

Fonctionnement. - Les bornes de sortie $\mathrm{E}_{1}$ et $\mathrm{E}_{2} \mathrm{du}$ thermocouple sont reliées aux bornes du galvanomètre. La résistance du cadre de celui-ci est grande devant celle du thermocouple. La f.é.m. $e$ engendrée produit dans le circuit un courant qui fait dévier le cadre.

Quand la régulation fonctionne, un faisceau lumineux issu d'une fente éclairée donne, après réflexion sur le miroir du cadre galvanométrique, un spot qui se forme sur la cellule différentielle, laquelle occupe une position $x_{0}$, fonction de la f.é.m. $e_{0}$, correspondant à une température $T_{q_{0}}$ de régulation souhaitée.

Lorsque $e$ est différent de $e_{0}$, les deux plages sont inégalement éclairées et un signal sinusoïdal d'amplitude variable provient du pont sous forme d'une tension égale à $\frac{\varepsilon V_{m}}{a} \cdot \cos \omega t$ avec $\varepsilon=x_{0}-x$ où $x$ est l'abscisse du centre du spot et $a$ une constante.

A la sortie du démodulateur apparaît une tension $U$ redressée qui est appliquée aux bornes de l'induit du moteur.

Le curseur du potentiomètre, entraîné par le rotor du moteur, fournit une tension $V$ communiquée au convertisseur de puissance chargé d'augmenter ou de diminuer la puissance rayonnante du fil de chauffage afin de porter le quartz à une température $T_{q}$ donnant $e$ égal à $e_{0}$. 
Fonctions de transfert opérationnelles du système régulateur/microbalance [2]. - La figure 2 donne le diagramme fonctionnel du système d'asservissement, similaire de celui d'un dispositif à tubes [5].

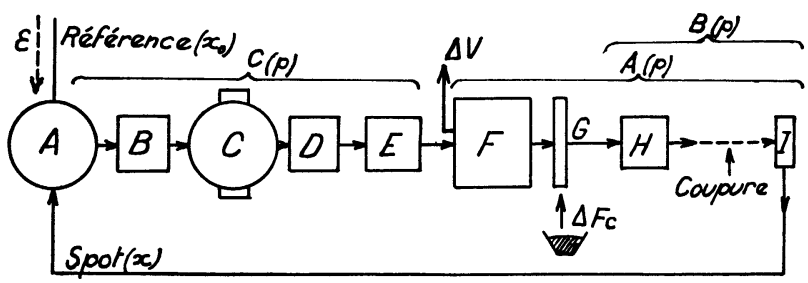

Frg. 2.

A : Détecteur d'écart. - B : Chaîne d'amplificateurs. - C : Servo-moteur. - D : Potentiomètre. - E : Correcteur de phase. - F : Étage de sortie. - G : Quartz. $\mathrm{H}$ : Thermocouple. - I : Galvanomètre.

a) La fonction de transfert de la chaîne ouverte est :

$$
K G(p)=A(p) C(p) .
$$

b) La fonction de transfert relative au déplacement du spot (abscisse : $x$ ) sous l'influence de $\Delta V$ et $\Delta F_{c}$ est :

$$
L(x)=A(p) L \Delta V+B(p) L \Delta F_{c}
$$

$\Delta V$ et $\Delta F_{c}$ étant respectivement les variations à partir de valeurs initiales de la tension de sortie du potentiomètre et du flux thermique reçu par le quartz.

Constitution et comportement du système électronique. - Le schéma de la figure 3 donne les éléments passifs et actifs composant le système électronique [4].

Les courbes de la figure 4 donnent les variations de la d.d.p. : $V_{\mathbf{E}}-V_{0}$, existant entre les bornes de sortie du démodulateur pour deux positions du spot symétriques par rapport au centre de la cellule.

Ainsi le moteur est amené à tourner dans un sens ou dans l'autre selon le signe de cette tension de sortie.

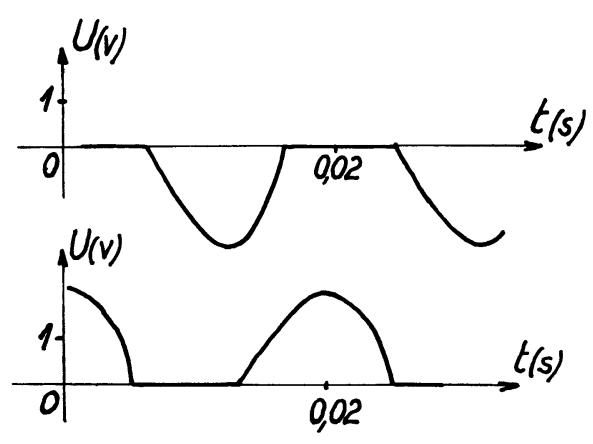

Fig. 4.

Stabilité. - La courbe (a) de la figure 5 représente la fonction de Nyquist, $K G(p)=A(p) C(p)$ dans le plan complexe avec $p=j \omega$, lorsque le régulateur fonctionne sans correcteur; la courbe (b) lorsqu'il fonctionne avec correcteur.

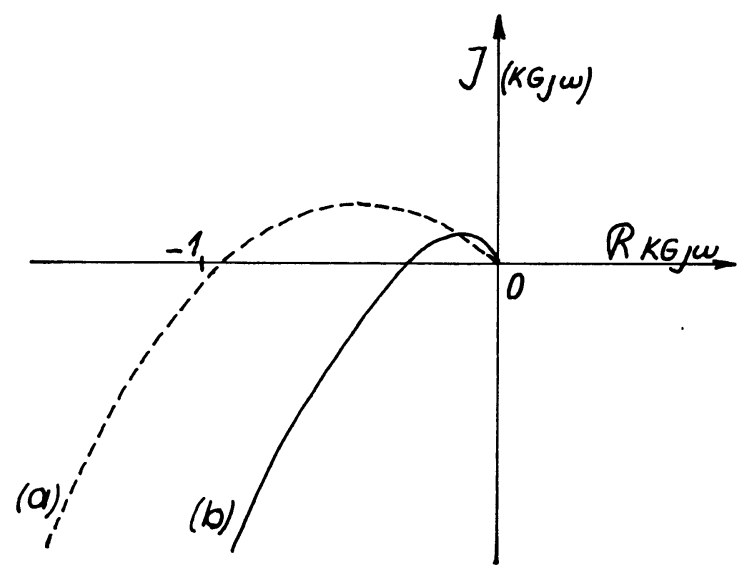

FIG. 5.

Elles sont relevées expérimentalement en appliquant entre les bornes du galvanomètre la tension :

$$
e=e_{0}+\Delta E_{0} \cos \omega t
$$

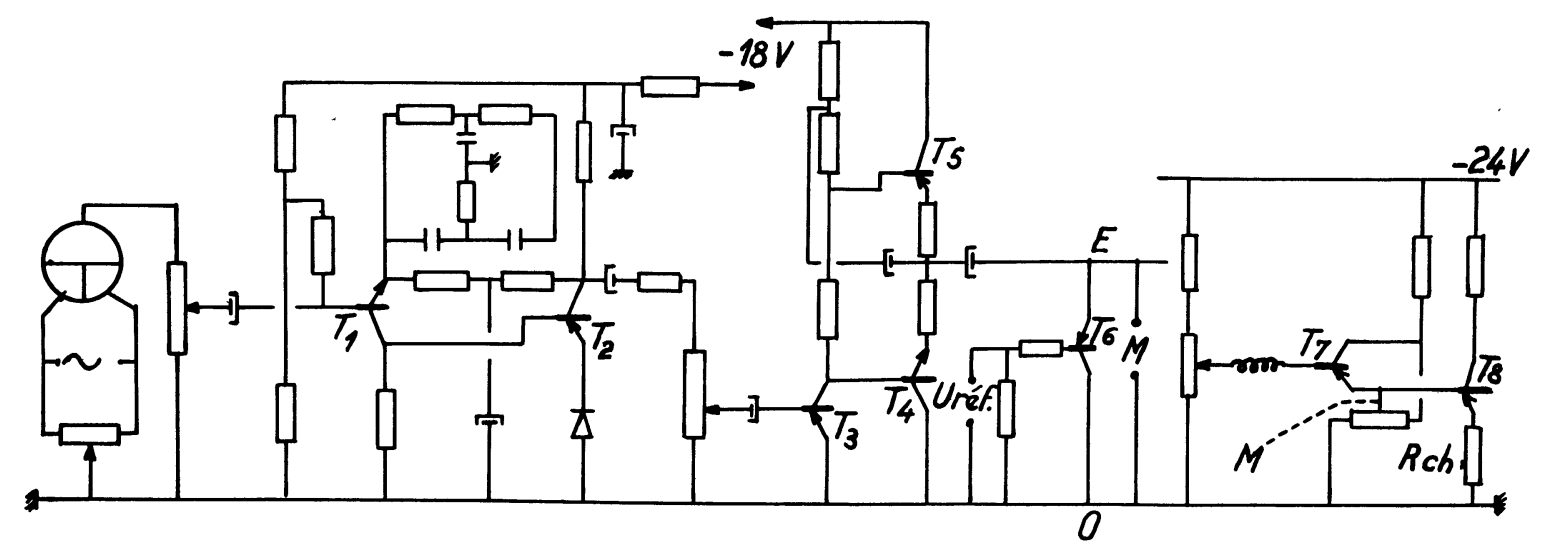

FIG. 3. 
et en recueillant entre les bornes de sortie du thermocouple la tension $e_{\mathrm{s}}=e_{1}+\Delta e_{0}|K G(j \omega)| \cos (\omega t+\varphi)$.

On constate que la courbe (b) révèle une marge de gain et une marge de phase suffisantes pour assurer un amortissement satisfaisant au régime transitoire.

Réponse du système à une perturbation extérieure. - a) En l'absence de perturbation sensible, on constate que $e$ reste égal à $e_{0}$ à $1 \mu \mathrm{V}$ près et la fréquence $F$ du quartz reste constante à 1 ou $2 \mathrm{~Hz}$ près;

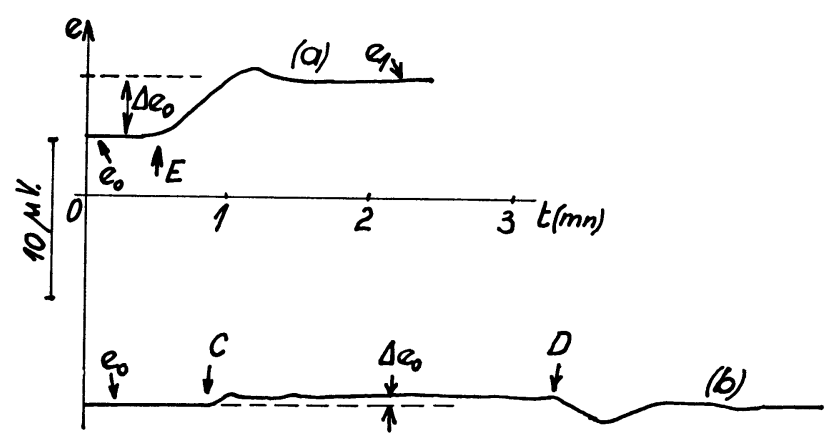

FIG. 6.

b) On déplace brusquement en $\mathrm{E}$ le chariot porteur de la cellule de façon telle que $e_{0}$ varie de $\Delta e_{0}$ (fig. 6). Des équations précédentes, on déduit :

$$
L x=\frac{A \cdot C}{1+A \cdot C} L x_{0} .
$$

On constate alors que $e$ variant, comme l'indique la courbe (a) de la figure 6, traduit un amortissement satisfaisant du régime transitoire. On observe alors qu'à l'état statique $x=x_{0}+\Delta x_{0}$, ou encore $e=e_{0}+\Delta e_{0}=e_{1}$

c) $e$ en l'absence de perturbation se maintenant constant, en $\mathrm{C}$, on découvre brusquement le creuset vide, porté à une température voisine de $1200^{\circ} \mathrm{C}$. Les équations précédentes donnent :

$$
L \varepsilon=-{ }_{1} \frac{B}{+A \cdot C} L \Delta F_{c}
$$

à l'état statique : $\varepsilon=0$ et $x=x_{0}$ et $\Delta V=K^{\prime} . \Delta F_{c}$ ( $K^{\prime}$ : constante).

En $D$, on masque brusquement le creuset. La courbe (b) de la figure 6 exprime alors une régulation convenable assurée par le servomécanisme.

La fréquence $F$ varie au plus de $15 \mathrm{~Hz}$ lorsque le creuset étant porté à $1200^{\circ} \mathrm{C}$, mais vide de matériau à évaporer, on passe d'un régime stationnaire où le creuset est masqué à un régime stationnaire où il est découvert.

Conclusion. - Ainsi, cet appareil permet de réguler au cours d'une évaporation la température d'un quartz vibrant à l'intérieur d'une plage telle qu'il n'en résulte qu'une perturbation négligeable sur la fréquence du quartz [4].

\section{BIBLIOGRAPHIE}

[1] Gouaulit (J.), C. R. Acad. Sc., 1966, 262, série B, 461 à 464.

[2] Gilife, Decaulne, Peiregrin, Théorie et calcul des asservissements, Dunod, 1958.
[3] Brevet C.N.R.S., PV 32.469.

[4] Additif au brevet C.N.R.S., PV 32.469.

[5] Gouault (J.), Thèse référence C.N.R.S., AO. 1127. 